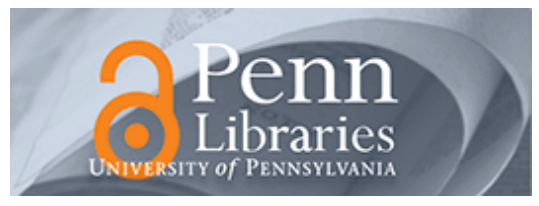

University of Pennsylvania

ScholarlyCommons

6-29-2007

\title{
Direct Transition From a Disordered to a Multiferroic Phase on a Triangular Lattice
}

Michel Kenzelmann

Gavin J. Lawes

A. Brooks Harris

University of Pennsylvania, harris@sas.upenn.edu

Goran Gašparović

Collin L. Broholm

See next page for additional authors

Follow this and additional works at: https://repository.upenn.edu/physics_papers

Part of the Physics Commons

\section{Recommended Citation}

Kenzelmann, M., Lawes, G. J., Harris, A., Gašparović, G., Broholm, C. L., Ramirez, A. P., Jorge, G. A., Jaime, M., Park, S., Huang, Q., Shapiro, A., \& Demianets, L. A. (2007). Direct Transition From a Disordered to a Multiferroic Phase on a Triangular Lattice. Physical Review Letters, 98 267205-1-267205-4.

http://dx.doi.org/10.1103/PhysRevLett.98.267205

This paper is posted at ScholarlyCommons. https://repository.upenn.edu/physics_papers/456

For more information, please contact repository@pobox.upenn.edu. 


\title{
Direct Transition From a Disordered to a Multiferroic Phase on a Triangular Lattice
}

\author{
Abstract \\ We report the first direct transition from a paramagnetic and paraelectric phase to an incommensurate \\ multiferroic in the triangular lattice antiferromagnet $\mathrm{RbFe}\left(\mathrm{MoO}_{4}\right)_{2}$. Ferroelectricity is observed only when \\ the magnetic structure has chirality and breaks inversion symmetry. A Landau expansion of symmetry- \\ allowed terms in the free energy demonstrates that chiral magnetic order can give rise to a pseudoelectric \\ field, whose temperature dependence agrees with experiment. \\ Disciplines \\ Physics \\ Author(s) \\ Michel Kenzelmann, Gavin J. Lawes, A. Brooks Harris, Goran Gašparović, Collin L. Broholm, Arthur P. \\ Ramirez, Guillermo A. Jorge, Marcelo Jaime, Sungil Park, Qingzhen Huang, Alex Ya Shapiro, and L. A. \\ Demianets
}




\title{
Direct Transition from a Disordered to a Multiferroic Phase on a Triangular Lattice
}

\author{
M. Kenzelmann, ${ }^{1,2,3}$ G. Lawes, ${ }^{4,5}$ A. B. Harris, ${ }^{6}$ G. Gasparovic,${ }^{2,3}$ C. Broholm, ${ }^{2,3}$ A. P. Ramirez,${ }^{4,7}$ G. A. Jorge, ${ }^{8}$ M. Jaime, ${ }^{8}$ \\ S. Park, ${ }^{3,9}$ Q. Huang, ${ }^{3}$ A. Ya. Shapiro, ${ }^{10}$ and L. A. Demianets ${ }^{10}$ \\ ${ }^{1}$ Laboratory for Solid State Physics, ETH Zurich, CH-8093 Zurich, Switzerland \\ ${ }^{2}$ Department of Physics and Astronomy, The Johns Hopkins University, Baltimore, Maryland 21218, USA \\ ${ }^{3}$ NIST Center for Neutron Research, National Institute of Standards and Technology, Gaithersburg, Maryland 20899, USA \\ ${ }^{4}$ Los Alamos National Laboratory, Los Alamos, New Mexico 87545, USA \\ ${ }^{5}$ Department of Physics and Astronomy, Wayne State University, Detroit, Michigan 48202, USA \\ ${ }^{6}$ Department of Physics and Astronomy, University of Pennsylvania, Philadelphia, Pennsylvania 19104, USA \\ ${ }^{7}$ Bell Laboratories, Alcatel-Lucent, Murray Hill, New Jersey 07974, USA \\ ${ }^{8}$ MPA-NHMFL, MS E536, Los Alamos National Laboratory, Los Alamos, New Mexico 87545, USA \\ ${ }^{9}$ HANARO Center, Korea Atomic Energy Research Institute, Daejeon, Korea \\ ${ }^{10}$ A. V. Shubnikov Institute for Crystallography RAS, 117333 Moscow, Russia
} (Received 14 February 2007; published 29 June 2007)

\begin{abstract}
We report the first direct transition from a paramagnetic and paraelectric phase to an incommensurate multiferroic in the triangular lattice antiferromagnet $\mathrm{RbFe}\left(\mathrm{MoO}_{4}\right)_{2}$. Ferroelectricity is observed only when the magnetic structure has chirality and breaks inversion symmetry. A Landau expansion of symmetry-allowed terms in the free energy demonstrates that chiral magnetic order can give rise to a pseudoelectric field, whose temperature dependence agrees with experiment.
\end{abstract}

DOI: 10.1103/PhysRevLett.98.267205

PACS numbers: 75.25.+z, 75.10.Jm, 75.40.Gb

Magnetic and electric degrees of freedom have different symmetry, and their coupling has intrigued scientists continually for over a hundred years [1,2]. Magnetic and electric orders compete on a local scale, making their coexistence relatively rare [3]. While traditional magnetoelectric multiferroics have separate magnetic and electric phase transitions [4], a new class of materials with simultaneous magnetic and ferroelectric phase transitions was recently discovered [5-7]. A common feature of these materials is the interaction between ferroelectric polarization and incommensurate magnetic order. Examples of such materials include the rare-earth $(R e)$ manganites $\left(R e \mathrm{MnO}_{3}\right.$ and $\left.\operatorname{Re} \mathrm{Mn}_{2} \mathrm{O}_{5}\right)$ [5,6,8] and $\mathrm{Ni}_{3} \mathrm{~V}_{2} \mathrm{O}_{8}$ [7].

Important insights into the microscopic origin of the magnetoelectric coupling were provided by a phenomenological theory which was based on a symmetry analysis of the magnetic and ferroelectric order parameters $[7,8]$ and established that the magnetoelectric coupling has a trilinear form. A number of microscopic theories have been proposed that respect these symmetry constraints for the known multiferroic materials [9-11]. The present results for $\mathrm{RbFe}\left(\mathrm{MoO}_{4}\right)_{2}$ (RFMO) provide a critical test for these theories of magnetoelectricity and reveal that incommensurability is not essential.

Single-crystal samples of RFMO were grown by a flux method [12]. RFMO is described by space group $P \overline{3} \mathrm{~m} 1$ at room temperature. At $T_{0}=180 \mathrm{~K}$, the symmetry is lowered to $P \overline{3}$ [13] by a lattice distortion with an amplitude that we denote by $\eta$. Figure 1(a) shows this structure which, though distorted, still preserves inversion symmetry and therefore is not ferroelectric. For $T<T_{0}$, RFMO contains perfect $\mathrm{Fe}^{3+}$ triangular lattice planes in which spins $S=5 / 2$ are coupled through antiferromagnetic superex- change interactions involving two oxygen anions. The magnetism is dominated by interactions within the plane with an energy scale of about $1 \mathrm{meV}$ [14]. The $\mathrm{Fe}^{3+}$ ions feature a sizable $X Y$ anisotropy [14], so that RFMO is essentially a $X Y$ antiferromagnet on a triangular lattice. The planes are coupled via at least 25 times weaker nearest- and next-nearest-neighbor interactions, inducing magnetic long-range order at $T_{N}=3.8 \mathrm{~K}$. Although RFMO contains perfect triangular $\mathrm{Fe}^{3+}$ lattices, Fig. 1(a) shows that out-of-plane ions lead to two types of triangles: "up triangles" with a green oxygen tetrahedron above the plane and "down triangles" with a tetrahedron below the plane. This feature of RFMO implies the absence of a mirror plane perpendicular to the $c$ axis, which is one of the necessary conditions for magnetoelectricity.

Neutron measurements were carried out using the BT2 and SPINS instruments at NIST and a high-field magnet with a dilution refrigerator. The zero-field magnetic order shown in Fig. 1(b) is characterized by incommensurate Bragg peaks at $\mathbf{Q}=\mathbf{G} \pm \mathbf{q}$, where $\mathbf{G}$ is a reciprocal lattice vector and $\mathbf{q}=\left(1 / 3,1 / 3, q_{z}\right)$ is the magnetic ordering wave vector in reciprocal lattice units. Figure 2(c) shows that $q_{z} \simeq 0.458$ for $T<T_{N}$ in zero field. The temperature dependence of the magnetic Bragg peak intensity, shown in Fig. 2(a), identifies the onset of magnetic order as a continuous phase transition for which representation theory [15] permits two types of magnetic order. The first type, strongly excluded by the diffraction data, has all spins parallel to the $c$ axis. The second type has all spins in the plane and is described by two complexvalued scalar order parameters $\sigma^{(n)}\left(q_{z}\right)$, with $n=1$, 2. In terms of these order parameters, the spin distribution is given by 


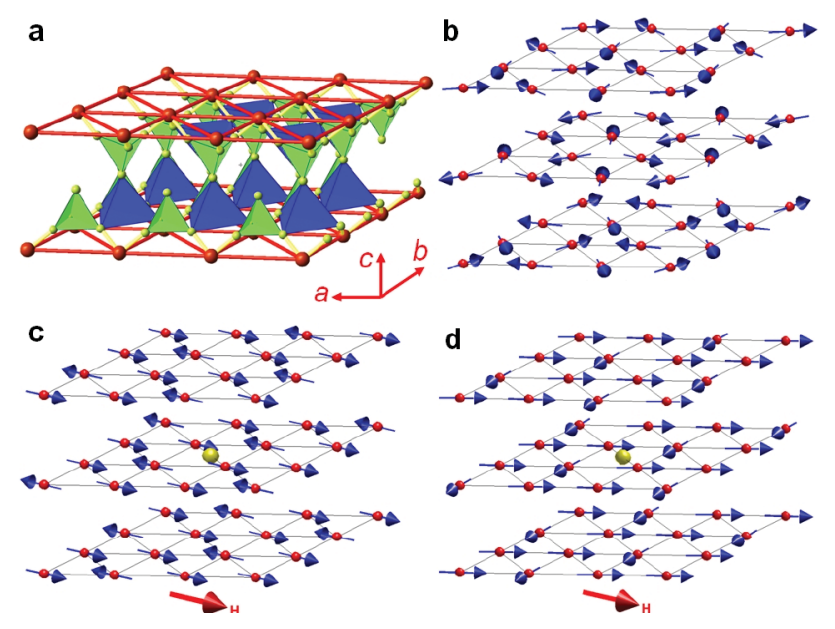

FIG. 1 (color). (a) Low-temperature chemical structure of RFMO, belonging to the trigonal space group $P \overline{3}$. Shown are the $\mathrm{Fe}^{3+}$ in red and $\mathrm{O}^{-2}$ ions in yellow. The $\mathrm{Fe}^{3+}$ are expected to interact through superexchange involving two $\mathrm{O}^{-2}$ for intraplane interactions and three $\mathrm{O}^{-2}$ for interplane interactions. There is one nearest-neighbor and two distinct next-nearest-neighbor interactions between planes. (b) Zero-field magnetic structure at $T=2 \mathrm{~K}$ with an ordered moment at each site $M=3.9(5) \mu_{B}$; (c) at $\mu_{0} H=6 \mathrm{~T}$ and $T=2 \mathrm{~K}$, two-thirds of the moments point with $M=3.8(3) \mu_{B}$ along the field direction and one-third with $M=2.8(3) \mu_{B}$ opposite to it; and (d) at $\mu_{0} H=10 \mathrm{~T}$ and $T=$ $0.1 \mathrm{~K}$, with $M=3.7(5) \mu_{B}$, one-third of the moments are perpendicular to the field and two-thirds are parallel to each other and form an angle of $30^{\circ}$ with the field direction. The structures shown in (c),(d) have an inversion center indicated by the yellow point.

$$
\begin{aligned}
& S_{x}(\mathbf{r})=\left[\sigma^{(1)}\left(q_{z}\right)+\sigma^{(2)}\left(q_{z}\right)\right] \exp (i \mathbf{q} \cdot \mathbf{r})+\text { c.c. } \\
& S_{y}(\mathbf{r})=i\left[\sigma^{(1)}\left(q_{z}\right)-\sigma^{(2)}\left(q_{z}\right)\right] \exp (i \mathbf{q} \cdot \mathbf{r})+\text { c.c., }
\end{aligned}
$$

where the out-of-plane component $q_{z}$ describes the interplanar spin rotation. At zero field at $T=2 \mathrm{~K}$, best agreement with diffraction intensities of 82 magnetic peaks ( $\chi^{2}=10.6$ and $R=0.11$ ) indicates that neighboring spins in the plane are rotated by $2 \pi / 3$ with respect to each other in a structure that is commonly referred to as the " $120^{\circ}$ structure." Here $\chi^{2}$ is the mean squared deviation between the model and the data in units of the variance, and $R=$ $(1 / N) \sum_{i}\left|I_{i}^{o}-I_{i}^{c}\right| / I_{i}^{o}\left(I_{i}^{o}\right.$ and $I_{i}^{c}$ are the observed and calculated intensities, respectively). Spins in adjacent planes are rotated by an angle $2 \pi q_{z}$.

The two order parameters $\sigma^{(1)}$ and $\sigma^{(2)}$ describe two structures which differ in the sign of the chirality. In RFMO, chirality is defined as follows: If, as one traverses clockwise the three vertices of up triangles [see Fig. 1(b)], the spin directions are rotated clockwise by $120^{\circ}$ $\left(-120^{\circ}\right)$, then the spin triangle is said to have positive (negative) chirality. The two structures represented by $\sigma^{(1)}$ and $\sigma^{(2)}$ are degenerate in energy, so there will be two domains in which the zero-field structures are described by either $\sigma^{(1)}=3.9(5)$ and $\sigma^{(2)}=0$ or $\sigma^{(1)}=0$ and $\sigma^{(2)}=$ $3.9(5)$ at $T=2 \mathrm{~K}$.

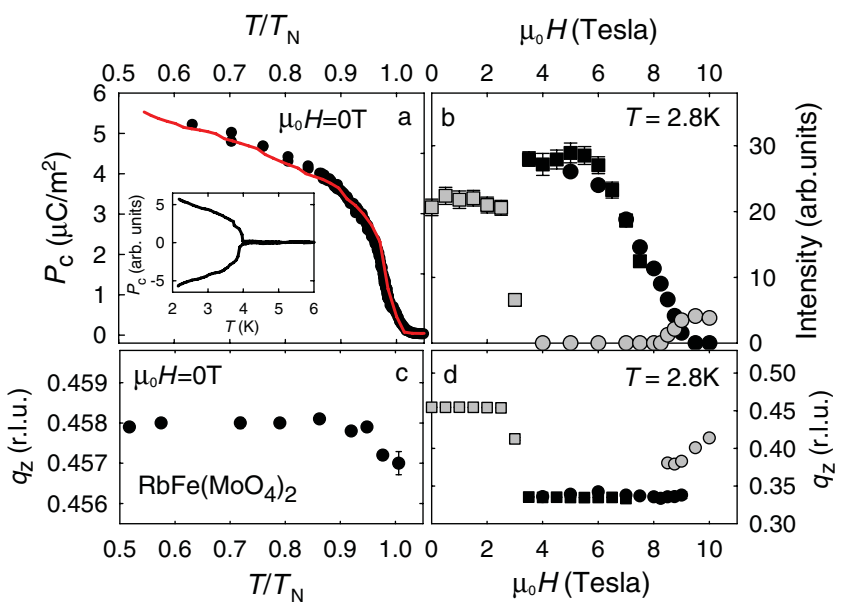

FIG. 2 (color). (a) Zero-field temperature dependence of the magnetic Bragg intensity (solid circles) observed at $\mathbf{Q}=$ $\left(1 / 3,1 / 3, q_{z}\right)$ compared to that of the ferroelectric polarization (solid line) $P_{c}$ along the $c$ axis. Inset: $P_{c}$ measured under positive and negative poling biases. (b) Field dependence of the intensity at $T=2.8 \mathrm{~K}$ for the commensurate (black) and the incommensurate (gray) reflections. The commensurate and the high-field incommensurate orders coexist at this temperature for a narrow field region between $\mu_{0} H=8$ and 9 T. (c),(d) Temperature and field dependence of $q_{z}$ at zero field and at $T=$ $2.8 \mathrm{~K}$, respectively. Squares and circles in (b), (d) distinguish two independent measurements, for which intensities were put on the same scale by matching data measured at the same field.

In-plane magnetic fields applied along the $[1,-1,0]$ direction lead to the collapse of the low-field incommensurate order in a discontinuous (first-order) phase transition for $\mu_{0} H=3.2 \mathrm{~T}$ at $T=2.8 \mathrm{~K}$ [see Fig. 2(b)]. For $3.2 \mathrm{~T}<$ $H<9.5 \mathrm{~T}$, commensurate order characterized by an ordering vector $\mathbf{q}=(1 / 3,1 / 3,1 / 3)$ is stabilized. For $\mu_{0} H \geq$ $9.5 \mathrm{~T}$, the magnetic order again becomes incommensurate, and the magnetic periodicity is noticeably field-dependent [see Fig. 2(d)]. The phase diagram in Fig. 3 was compiled from the temperature and field dependence of magnetic Bragg peaks and by tracking specific heat and dielectric anomalies. In the present Letter, we attempt to describe and understand the properties of the low-field phases below $\mu_{0} H=10 \mathrm{~T}$.

Symmetry requires that at nonzero field the magnetic structures in RFMO contain both order parameters $\sigma^{(1)}$ and $\sigma^{(2)}$ of opposite chirality. At $\mu_{0} H=6 \mathrm{~T}$ and $T=2.8 \mathrm{~K}$, best agreement with diffraction intensities of 62 magnetic peaks ( $\chi^{2}=5.2$ and $R=0.14$ ) is found for the magnetic structure shown in Fig. 1(c) that is given by $\sigma^{(1)}=$ $[1.09(4)+i 1.85]$ and $\sigma^{(2)}=[1.09(4)+i 1.94(4)]$ (error bars are provided only for free parameters) and where the magnitude of the ordered moments are $3.8(3) \mu_{B}$ and 2.8(3) $\mu_{B}$ along and opposite to the magnetic field, respectively. For the magnetic structure at $\mu_{0} H=10 \mathrm{~T}$ and $T=$ $0.1 \mathrm{~K}$, where we have measured only 15 magnetic peaks, we imposed an equal moment magnitude condition. Here the best agreement with diffraction data $\left(\chi^{2}=22.6\right.$ and 


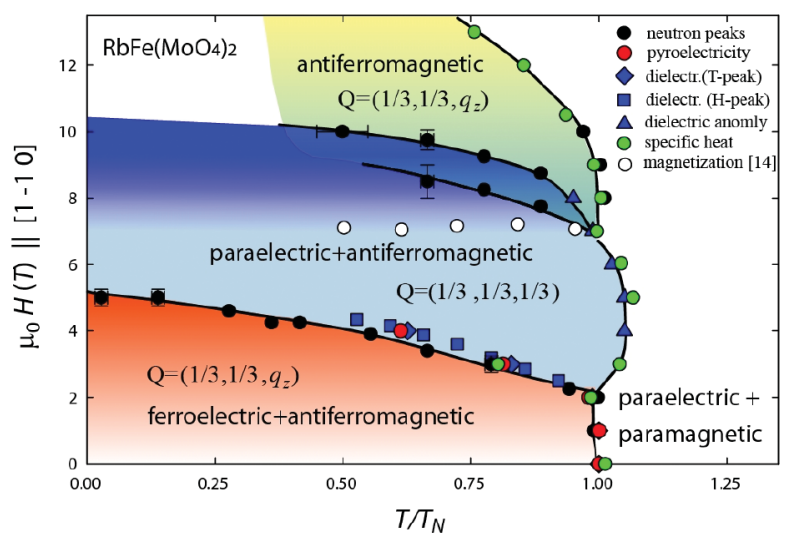

FIG. 3 (color). HT phase diagram of RFMO, for fields applied along the $[1,-1,0]$ direction with $T_{N} \approx 3.8 \mathrm{~K}$, obtained through various techniques. Open circles represent the end of a magnetization plateau as a function of field [14]. The color gradient indicates the estimated increasing magnetization as a function of magnetic field. The boundary of the high-field incommensurate phase has not been determined for $T<2 \mathrm{~K} \sim 0.5 T_{N}$. Subtle phase modifications which may occur in small regions adjacent to the $\mathbf{Q}=(1 / 3,1 / 3,1 / 3)$ phase [14] are not shown. The commensurate and the high-field incommensurate magnetic orders coexist in a narrow field region for $\mu_{0} H \approx 9 \mathrm{~T}$. The two solid lines indicate where each type of order was observed in diffraction data [see Figs. 2(b) and 2(d)].

$R=0.21)$ was found for the magnetic structure shown in Fig. 1(d) and is given by $\sigma^{(1)}=[2.16(3)+i 0.197]$ and $\sigma^{(2)}=[-1.251-i 1.724]$. Figures $1(\mathrm{~b})-1(\mathrm{~d})$ show that the zero-field structure features a chiral order parameter, while both commensurate structures contain a point of inversion.

The absence of a point of inversion for the low-field magnetic structure prompted us to examine the dielectric properties of the material. The pyroelectric current was measured at fixed $H$ and the magnetoelectric current at fixed $T$, using a Keithley electrometer. Figure 2(a) shows the development of ferroelectric polarization along the $c$ axis upon cooling the sample in zero field. The electric polarization can be switched by applying an opposite poling bias as shown in the inset in Fig. 2(a), demonstrating that RFMO is a ferroelectric below $T_{N}$. Only a single peak was observed in the temperature dependence of the specific heat at zero field measured using a $2 \mathrm{mg}$ single-crystal sample with a $\mathrm{SiN}$ thin-membrane microcalorimeter [16], demonstrating that the ferroelectric transition coincides with the onset of antiferromagnetic order. The polarization is reduced at small magnetic fields and completely suppressed in the commensurate phase. Further, we observed small anomalies in the dielectric constant at the commensurate phase boundary. These measurements identify the low-field phase as ferroelectric and the field-induced phases as paraelectric.

In multiferroics such as $\mathrm{TbMnO}_{3}$ and $\mathrm{Ni}_{3} \mathrm{~V}_{2} \mathrm{O}_{8}$, a ferroelectric order parameter appears when inversion symmetry is broken by the magnetic structure $[7,8]$. This occurs through two consecutive continuous phase transitions, each characterized by the appearance of a magnetic order parameter. A Landau theory $[7,8]$ which couples the two magnetic and one ferroelectric order parameters successfully describes the direction of the ferroelectric polarization, demonstrating that magnetic order induces ferroelectricity. Ferroelectricity in these systems may also be understood by the spin-current interaction, which is due to superexchange and the spin-orbit interactions [9], giving the spontaneous electric polarization that arises from canted spins on neighboring sites $i$ and $j$ as $\mathbf{P}_{i j}=\left[\mathbf{S}\left(\mathbf{r}_{i}\right) \times\right.$ $\left.\mathbf{S}\left(\mathbf{r}_{j}\right)\right] \times\left[\mathbf{r}_{i}-\mathbf{r}_{j}\right]$. For RFMO, $\mathbf{P}_{i j}$ lies in the basal plane, and, in view of the threefold rotation axis, the macroscopic polarization $P=\sum_{\langle i j\rangle} \mathbf{P}_{i j}$ vanishes. RFMO does exhibit ferroelectricity, so the spin-current interaction alone [9], while possibly valid for some systems, does not provide a general explanation for magnetoelectricity. In contrast, we shall show that the symmetry-based phenomenological theory $[7,8,17]$ accurately describes magnetoelectricity in RFMO.

Here we will explicitly consider the Landau theory only for the case of zero magnetic field, although the general conclusions are expected to remain valid until a phase boundary is crossed. The free energy is invariant under the symmetries of the magnetically disordered phase, which include $\mathcal{T}$, time reversal $t \rightarrow-t, I$, spatial inversion $\mathbf{r} \rightarrow-\mathbf{r}$, and $\mathcal{R}_{3}$, the threefold rotation about the $c$ axis. Since the effect of these operations on $\mathbf{S}(\mathbf{r})$ is known, one can deduce the effect of these operations on the order parameters. Thus, using Eq. (1), we find that

$$
\begin{gathered}
\mathcal{T} \sigma^{(n)}\left(q_{z}\right)=-\sigma^{(n)}\left(q_{z}\right), \quad I \sigma^{(1)}\left(q_{z}\right)=\sigma^{(2)}\left(q_{z}\right)^{*}, \\
\mathcal{R}_{3} \sigma^{(n)}\left(q_{z}\right)=e^{2 n \pi i / 3} \sigma^{(n)}\left(q_{z}\right) .
\end{gathered}
$$

Taking account of these symmetries, one sees that the free energy must assume the form

$$
F=\frac{1}{2} \sum_{n, \pm} r\left( \pm q_{z}\right)\left|\sigma^{(n)}\left( \pm q_{z}\right)\right|^{2}+\mathcal{O}\left(\sigma^{4}\right)
$$

where $r\left(q_{z}\right)$ is an even function of $q_{z}$ in the $P \overline{3} m 1$ space group above $T_{0}=180 \mathrm{~K}$ but develops a contribution proportional to $\eta q_{z}$ in the $P \overline{3}$ space group below $T_{0}$. Minimization of $F$ shows that $1 / 2-q_{z}$ is proportional to the lattice distortion $\eta$ away from the $P \overline{3} m 1$ space group. The transition at $T_{0}=180 \mathrm{~K}$ does not break inversion symmetry, but it does determine the incommensurate wave vector $q_{z}$. As Eq. (3) indicates, the lattice distortion actually selects the helicity (the sign of $q_{z}$ ), although it does not lift the degeneracy between magnetic structures with opposite chirality $\sigma^{(1)}$ and $\sigma^{(2)}$.

At zero field, the effect of the terms in the free energy of order $\sigma^{4}$ is to prevent $\sigma^{(1)}$ and $\sigma^{(2)}$ from simultaneously being nonzero. We now consider the lowest order terms allowed by symmetry which couple the magnetic order parameters to the spontaneous polarization $\mathbf{P}$. The lowest order interaction that is consistent with the symmetries of 
Eq. (2), induces a spontaneous polarization, and also conserves wave vector must be of the form $V=\sum_{n m} c_{n m}^{\gamma} \times$ $\sigma^{(n)}\left(q_{z}\right) \sigma^{(m)}\left(q_{z}\right)^{\star} P_{\gamma}$, where $\gamma$ labels the Cartesian component of the polarization. For the magnetoelectric interaction $V$, invariance under $I$ implies that $c_{n m}^{\gamma}$ is nonzero only for $n=m$ and $c_{11}^{\gamma}=-c_{22}^{\gamma}$. Furthermore, since $\left|\sigma^{(n)}\left(q_{z}\right)\right|^{2}$ is invariant under the threefold rotation about the $c$ axis $\mathcal{R}_{3}$, one must have $\mathcal{R}_{3} P_{\gamma}=P_{\gamma}$. Thus, the magnetoelectric free energy in RFMO assumes the form

$$
F=\frac{1}{2} \chi^{-1} P^{2}+K\left[\left|\sigma^{(1)}\left(q_{z}\right)\right|^{2}-\left|\sigma^{(2)}\left(q_{z}\right)\right|^{2}\right] P_{c},
$$

where $\chi$ is the electric susceptibility, which we have measured to be $\chi \approx 4(1) \epsilon_{0} \approx 4(1) \times 10^{-11} \mathrm{C}^{2} / \mathrm{J} \mathrm{m}$ away from the phase boundaries, and the value of the coefficient $K$ is not fixed by symmetry. In contrast to the situation in some of the previously studied oxides [5-8], the present results show that ferroelectricity induced by magnetism does not require the simultaneous presence of two separate magnetic order parameters that are critical at distinct continuous phase transitions.

An advantage of the symmetry-based phenomenological theory is its close connection to microscopic models, such as the magnetoelastic theory developed for $\mathrm{Ni}_{3} \mathrm{~V}_{2} \mathrm{O}_{8}$ [11]. At low temperature, Fig. 2(a) shows an electric polarization $P \approx 5 \times 10^{-6} \mathrm{C} / \mathrm{m}^{2}$ from which we deduce that $K \approx$ $2 \times 10^{5} \mathrm{~V} / \mathrm{m}$. This value of $P$ corresponds to a ferroelectric atomic displacement (probably of the oxygen ions) of order $10^{-5} \AA$. The trilinear interaction suggests that ferroelectricity could be induced by a microscopic interaction of the form $V \sim\left(\partial J / \partial u_{i j}\right) u_{i j} S_{\alpha}(i) S_{\beta}(j)$, where $J_{\alpha \beta}$ is an exchange tensor. The value of $K$ corresponds to $\partial J / \partial u$ of order $10^{-4} \mathrm{eV} / \AA$ for $\alpha \neq \beta$.

When the free energy of Eq. (4) is minimized with respect to $P_{c}$, one finds its equilibrium value $\left\langle P_{c}\right\rangle$ to be proportional to $\left|\sigma^{(1)}\right|^{2}-\left|\sigma^{(2)}\right|^{2}$. So $P_{c}$ is nonzero as long as the net chirality on up triangles $\left|\sigma^{(1)}\right|^{2}-\left|\sigma^{(2)}\right|^{2}$ is nonzero. At zero field, only one of the two magnetic order parameters $\sigma^{(i)}$ is nonzero, so the temperature dependence of the magnetic Bragg peak, which is proportional to $\left|\sigma^{(i)}\right|^{2}$, is predicted by this theory to exactly match that of the ferroelectric polarization. The excellent agreement of the data reported in Fig. 2(a) with this prediction provides strong support for the validity of the theory. The fact that $P_{c}$ couples to a difference in order parameters suggests that applying an electric field to an untwinned sample at zero magnetic field would favor a magnetic structure with definite chirality on up triangles described by $\sigma^{(1)}$ or $\sigma^{(2)}$. Furthermore, because there is a definite helicity associated with the incommensurate modulation, an untwinned sample in an electric field should also be helical.

Both commensurate spin structures at 6 and $10 \mathrm{~T}$ have $\left|\sigma^{(1)}\right|^{2}=\left|\sigma^{(2)}\right|^{2}$ within error bars, so there can be no magnetoelectric coupling in that phase-consistent with the observed absence of ferroelectricity. Equation (4) further implies that the high-field incommensurate phase is ferroelectrically polarized along the $c$ axis if $\left|\sigma^{(1)}\right|^{2} \neq$ $\left|\sigma^{(2)}\right|^{2}$ and paraelectric otherwise. We remark that incommensurability is not a requirement for magnetoelectricity in RFMO, possibly similar to some of the manganite perovskites [18]. Indeed, we claim that, even without the $P \overline{3} m 1 \rightarrow P \overline{3}$ lattice distortion, the resulting commensurate magnetic structure would still be ferroelectric. This can be appreciated through Landau theory or through the following intuitive argument. To induce a ferroelectric polarization, a magnetic structure must break inversion symmetry and define a unique direction. Figure 1(b) shows that the $120^{\circ}$ structure clearly breaks inversion symmetry. The magnetic structure also defines a unique direction, because triangles with positive chirality define a unique direction given by the direction from such a triangle to the nearest oxygen tetrahedron [green triangles in Fig. 1(a)]. So, while the absence of a mirror plane perpendicular to the $c$ axis is an essential ingredient of magnetoelectricity, incommensurability surprisingly is not.

In summary, the novel multiferroic RFMO with a simultaneous onset of magnetic and ferroelectric order offers the simplest case thus far wherein a phenomenological trilinear coupling theory accurately describes the symmetry properties of magnetic and ferroelectric order. Our approach allows quantitative comparisons between theory and experiment and leads to the astonishingly general prediction that trigonal stacked triangular antiferromagnets with a $120^{\circ}$ spin structure are multiferroic.

C. B. acknowledges useful discussions with O. Tchernyshyov and D. Clarke. Work at ETH was supported by the Swiss NSF under Contract No. PP002102831. Work performed at the NHMFL is supported by the NSF, the DOE, and the State of Florida. Work at the Shubnikov Institute was supported by the U.S. Civilian Research \& Development Foundation Grant No. RP12097. Work at JHU and at SPINS was supported by the NSF through Grants No. DMR-0306940 and No. DMR9986442, respectively.

[1] P. Curie, J. Phys. Theor. Appl. 3, 393 (1894).

[2] M. Fiebig, J. Phys. D: Appl. Phys. 38, R123 (2005).

[3] N. A. Hill et al., J. Magn. Magn. Mater. 242, 976 (2002).

[4] G. A. Smolenskii et al., Sov. Phys. Usp. 25, 475 (1982).

[5] T. Kimura et al., Nature (London) 426, 55 (2003).

[6] N. Hur et al., Nature (London) 429, 392 (2004).

[7] G. Lawes et al., Phys. Rev. Lett. 95, 087205 (2005).

[8] M. Kenzelmann et al., Phys. Rev. Lett. 95, 087206 (2005).

[9] H. Katsura et al., Phys. Rev. Lett. 95, 057205 (2005).

[10] I. A. Sergienko et al., Phys. Rev. B 73, 094434 (2006).

[11] A. B. Harris et al., Phys. Rev. B 73, 184433 (2006).

[12] R. F. Klevtsova et al., Kristallografiya 15, 209 (1970).

[13] G. Gasparovic, Ph.D. thesis, Johns Hopkins University.

[14] L. E. Svistov et al., Phys. Rev. B 67, 094434 (2003).

[15] A. B. Harris, arXiv:cond-mat/0610241.

[16] G. A. Jorge et al., Physica (Amsterdam) 354B, 297 (2004).

[17] M. Kenzelmann et al., Phys. Rev. B 74, 014429 (2006).

[18] N. Aliouane et al., Phys. Rev. B 73, 020102(R) (2006). 